Loading

The Journal of the Canadian Game Studies Association

\title{
Indies de province
}

Deux développeurs de jeux indépendants dans le Québec

d'avant Internet

\section{Indies de province}

\section{Two independent game developers in pre-Internet Quebec}

\section{Jonathan Lessard and Carl Therrien}

Volume 14, Number 23, 2021

Le jeu vidéo au Québec

The Video Game in Quebec

URI: https://id.erudit.org/iderudit/1078726ar

DOI: https://doi.org/10.7202/1078726ar

See table of contents

Publisher(s)

Canadian Game Studies Association

ISSN

1923-2691 (digital)

Explore this journal

Cite this article

Lessard, J. \& Therrien, C. (2021). Indies de province : deux développeurs de jeux indépendants dans le Québec d'avant Internet. Loading, 14(23), 15-35.

https://doi.org/10.7202/1078726ar
Article abstract

This paper looks at the emergence of video game creation in Québec prior to the industrial boom and the popularization of independent games. Built from personal archives and oral history, the paper highlights two unknown personalities from the history of video games in Québec: Christian Boutin and I-Grec. These portraits contribute to diversify the "indie" narrative and reconsider it as part of a longer history.
Copyright (c) Jonathan Lessard, Carl Therrien, 2021

(c) (i) (5)
This document is protected by copyright law. Use of the services of Érudit (including reproduction) is subject to its terms and conditions, which can be viewed online.

https://apropos.erudit.org/en/users/policy-on-use/ 


\title{
Indies de province : deux développeurs de jeux indépendants dans le Québec d'avant Internet
}

\section{Indies de province : two independent game developers in pre-Internet Quebec}

\author{
Jonathan Lessard \\ Université Concordia \\ jonathan.lessard@concordia.ca \\ Carl Therrien \\ Université de Montréal \\ carl.therrien@umontreal.ca
}

\begin{abstract}
Résumé
Cet article s'intéresse à l'émergence de la création vidéoludique au Québec avant le boom industriel et la popularisation du jeu indépendant. À partir d'archives et d'entretiens avec deux concepteurs autodidactes ayant œuvré dans les années 1990, l'article propose un portrait de deux personnalités méconnues de l'histoire québécoise du jeu : Christian Boutin et I-Grec. Ces portraits permettent de diversifier le récit du phénomène indie et de le réinsérer dans une histoire de plus longue durée.
\end{abstract}

\section{Mots-clés}

Histoire, jeu, Québec, indépendant, partagiciel.

\begin{abstract}
This paper looks at the emergence of video game creation in Québec prior to the industrial boom and the popularization of independent games. Built from personal archives and oral history, the paper highlights two unknown personalities from the history of video games in Québec: Christian Boutin and I-Grec. These portraits contribute to diversify the "indie" narrative and reconsider it as part of a longer history.
\end{abstract}

\section{Keywords}

History, game, Québec, independent, shareware. 


\section{Indie - une contreculture ${ }^{1}$ vidéoludique mondialisée}

\section{Jeunes et citoyens du monde}

Désormais bien établi comme phénomène culturel de masse, le jeu vidéo voit s'affirmer en son sein, depuis une quinzaine d'années, un mouvement en réaction aux productions dominantes. À l'instar de la musique et du cinéma, il existe désormais une scène dite indépendante ou alternative (indie pour les intimes) du jeu vidéo. Cette scène et ses acteurs - comme pour plusieurs autres mouvements culturels - ne sont pas strictement définis ; on trouve de nombreux points de vue, parfois contradictoires, sur ce que signifie ou devrait signifier «être indie ». La constante est qu'une production indie s'écarte de la norme établie par les entreprises ou les titres dominants au moment où elle voit le jour. Cet écart peut se marquer dans les moyens de production (petites équipes, peu ou pas de financement), dans l'esthétisme (design en rupture avec les canons) ou par des critères éthiques (volonté de tenir un discours politique ou de ne pas satisfaire aux critères de succès commerciaux).

Si le Québec est présentement un leader international dans le développement du jeu vidéo dit mainstream, son écosystème de développeurs indie n'est pas en reste. Les Ubisoft, Electronic Arts, Warner Bros. et autres grands studios défrayent plus souvent la manchette, mais une ribambelle de petits et de moyens studios indépendants, tels que Borealys, Ko-Op, Behaviour, Outerminds et plusieurs autres, ont connu tantôt des succès financiers (souvent grâce à des campagnes de sociofinancement réussies), tantôt cette reconnaissance internationale allant de pair avec l'obtention de prix prestigieux - et parfois les deux. Le jeu Fez (2012) de Polytron, notamment, a vendu plus de un million de copies et obtenu, entre autres, le grand prix de 1'Independent Games Festival ${ }^{2}$. La majorité de ces entreprises sont rassemblées sous l'égide de la Guilde du jeu vidéo du Québec, qui se définissait à l'origine comme « la plus grosse coopérative de jeux vidéo indépendants au monde $»^{3}$.

Dans Handmade Pixels, Jesper Juul (2019) argue que l'éthique du mouvement indie est liée à une quête d'authenticité — ou du moins à une rhétorique de l'authenticité. Les jeux indépendants sont faits par de petites équipes qui n'ont pas, comme les grandes entreprises, à répondre à des actionnaires ou au service marketing. Ces équipes seraient libres de faire les choses comme elles le veulent et leurs œuvres auraient donc quelque chose de vrai, contrairement aux produits manufacturés par les grands studios. On pourrait donc s'attendre à un particularisme régional plus marqué de la part des développeurs indépendants que des grands studios. C'est ce qui s'observe dans la plupart des autres courants dits alternatifs : cuisines ultra-locales, cinémas nationaux, scènes musicales associées à des villes, voire à des quartiers. Or, Felan Parker et Jennifer Jensen démontrent qu'il est tout aussi difficile d'attribuer une certaine "canadienneté » aux jeux indépendants qu'aux blockbusters produits au pays cela vaut pour la production québécoise (Parker et Jenson, 2017) ${ }^{4}$. Bien sûr, les facteurs locaux jouent un rôle ; par exemple, Parker et Jensen soulignent que le coût de la vie relativement faible à Montréal favorise les développeurs indépendants, qui peuvent étirer leurs petits budgets sur une plus longue période que ceux établis à Toronto, à Vancouver ou à Los Angeles (p. 878). Mais si ces facteurs influent sur les modes de production, ils semblent sans incidence sur les contenus destinés au marché international. 


\section{"Vintage » et local}

Dans cet article, nous aimerions remettre en question l'idée que la scène indie internationale serait ultra-contemporaine. En premier lieu, les créateurs n'ont pas attendu l'émergence de cette scène pour concevoir des jeux de façon indépendante. Au contraire, les travaux de Melanie Swalwell rappellent que la programmation - et la programmation de jeux en particulier était au cœur des pratiques des premiers utilisateurs d'ordinateurs personnels (Swalwell, 2008). Plutôt qu'un phénomène nouveau, l'éthique de la vague indie — « faire des jeux soi-même »constituerait en quelque sorte une reprise des modalités premières de programmation en microinformatique.

D'autre part, les jeux d'ordinateur (et l'informatique en général), en tant que premiers objets culturels natifs du numérique, ont été d'emblée internationalisés. Même en l'absence d'un Internet mondialisé, les logiciels ont toujours été très faciles à dupliquer et à disséminer, ce dont ne se sont pas privés les utilisateurs mis en appétit par la rareté des produits. Contrairement à la musique, aux films et aux livres, dont la diffusion est depuis longtemps contrôlée par des réseaux d'éditeurs et de distributeurs, les premières générations de jeux d'ordinateur circulaient librement parmi les communautés d'adeptes de la première heure, où avait cours la copie illégale aussi bien que légale.

Prenant du recul par rapport au nouveau et à l'international, nous nous intéresserons à l'ancien et au local. À l'ère de la culture mondialisée, c'est souvent ce qui se retrouve en mal de documents. Les jeux vidéo connaissent une obsolescence extrêmement rapide. La nécessité de faire connaitre sans cesse de nouveaux jeux relègue aux oubliettes les anciens. Ayant été publicisés par magazines papier ou babillards électroniques, et même s'ils ont laissé des traces dans quelques pages de magazines électroniques — et encore, pour combien de temps? plusieurs jeux diffusés avant Internet sont très peu présents sur le Web. Quant à l'origine des œuvres, elle fait rarement partie des stratégies de communication. Il arrive aux membres les plus actifs de la communauté d'apprendre avec surprise qu'un de leurs jeux préférés a été développé à quelques coins de rue de chez eux.

Nous tenterons de faire œuvre utile en dressant ici le portrait de deux développeurs québécois que l'on pourrait anachroniquement qualifier d'indépendants et dont la visibilité sur Internet (et la notoriété en général) est quasiment nulle. Ce faisant, nous verserons des documents au compte d'une histoire mal connue, celle des pionniers de la culture numérique au Québec, et particulièrement de ses marges : les fameux «programmeurs de sous-sol» fantasmés. Les trajectoires de Christian Boutin (Oracle Software) et d'I-Grec (qui préfère ne pas divulguer son vrai nom) nous permettront de mieux comprendre les pratiques des développeurs de jeux indépendants québécois durant les années 1990, de nous faire une idée plus juste du contexte dans lequel elles s'inscrivent, mais également de diversifier le récit du mouvement indie contemporain et de l'insérer dans une histoire de plus longue durée. En filigrane, nous nous poserons de même la question de la québécité : est-ce que les dimensions culturelle et géographique avaient une plus grande incidence sur le développement de jeux indépendants (qu'il s'agisse de la production ou des contenus) avant l'avènement d'un Internet mondialisé ?

Les courtes biographies proposées ici sont le fruit d'une entrevue d'environ deux heures avec chacun des développeurs, mais aussi d'un engagement direct avec leur portfolio de jeux. Les 
questionnaires d'entrevue ont été développés et personnalisés à partir de cette recherche préalable. Bien sûr, ce genre d'histoire orale comporte des risques d'inexactitudes. Les témoignages constituent une reconstitution a posteriori des faits. Nous ne pouvons évidemment pas vérifier l'état d'esprit des concepteurs, non plus que les rapports qu'ils entretenaient avec leurs œuvres à l'époque qui nous intéresse. Nous nous contenterons donc de rapporter leurs témoignages, de les mettre en rapport avec les documents concrets tirés de notre fonds d'archives, et laisserons le lecteur juge de nos interprétations.

Nous commencerons par une présentation générale des deux artistes du jeu vidéo, leurs débuts étant caractérisés par plusieurs similarités. Nous nous pencherons ensuite sur leurs trajectoires respectives, qui divergent du point de vue des ambitions tant commerciales qu'esthétiques.

\section{Jouer et programmer}

\section{Les premiers enfants du numérique?}

On considère généralement que les «enfants du numérique » sont nés à partir de la fin des années 1980. Certains auraient toutefois pris de l'avance. Christian Boutin naît à Amos en 1974 et I-Grec à Granby en 1976, soit juste avant que les premiers micro-ordinateurs principalement le TRS-80 et l'Apple II (lancés en 1977) — ne soient disponibles sur le marché de masse nord-américain. Tous deux ont la chance d'être non seulement exposés à l'informatique dans leur jeunesse, mais également de s'y intéresser. Ce qui est loin d'aller de soi quand on considère que ces machines représentaient essentiellement des télévisions hors de prix, qui n'affichaient que du texte ou des graphismes abstraits, et qu'il fallait apprendre par cœur des commandes compliquées pour en tirer un début de quelque chose. Comme le résume Christian, «il n'y avait à peu près rien d'utile à faire à la maison avec un ordinateur à l'époque ». Rappelons que ces machines ne sont connectées à aucun réseau et sont livrées sans aucun logiciel de base, pas même un traitement de texte.

Pour Christian, le vecteur d'attractivité, ce sont les jeux vidéo qui font simultanément leur apparition dans les foyers. Il raconte avoir fréquenté assidument les pool-rooms enfumés d'Amos pour jouer aux bornes d'arcade ou regarder d'autres joueurs. Comme la plupart des enfants de son âge à l'époque, il voulait avoir à la maison une console de jeu comme l'Atari 2600, mais ses parents refusent. En guise de compromis, ils acquièrent un TRS-80 en 1982 (alors qu'il n'a que huit ans). Non sans prévoyance, ils considèrent que l'ordinateur (même s'il peut servir de console de jeu dans une certaine mesure) a au moins une valeur éducative : il est susceptible de préparer leurs enfants à un monde où l'informatique risque de jouer un rôle de plus en plus important.

I-Grec devra attendre ses douze ans (1988) pour qu'un ordinateur — un Tandy 1000 — fasse son entrée chez lui, et pour des raisons identiques : « Non aux consoles, OK pour l'ordinateur ». Il avait été exposé plus tôt à l'informatique grâce à son grand-père qui, pour son travail, disposait d'un ordinateur portable sur lequel I-Grec a pu découvrir ses premiers jeux : « Mon grand-père s'était acheté en 1986 un laptop. Un fucking laptop! C'est lui qui m'a montré comment ça marchait. » Il se rappelle aussi avoir vu traîner un ordinateur sur chariot à roulettes, dans les couloirs de son école primaire de Granby, dont personne ne se servait: «Les professeurs ne savaient pas quoi faire avec ça ». Il aurait obtenu la permission de s'en servir une heure par semaine. 
Il n'est pas nécessaire d'avoir grandi avec un ordinateur pour faire des jeux vidéo. Lorsque Christian et I-Grec découvrent l'informatique, d'autres avant eux avaient déjà conçu des jeux d'arcade, de console ou d'ordinateur. La différence, c'est que leur génération sera la première à aborder la conception de jeu avec pour principal bagage l'expérience d'autres jeux. La génération précédente, les fondateurs des compagnies phares de l'époque, comme Atari pour les consoles ou Sierra pour l'ordinateur, s'étaient mis à cette pratique en tant qu'adultes, l'abordant à partir de références culturelles pré-numériques.

Malgré leur jeune âge et le fait qu'ils évoluent dans des petites villes périphériques, Christian et I-Grec ont déjà accès à la plus grande partie de ce qui se fait de significatif sur ordinateur. D'une part, il n'y a pas tant de logiciels et de jeux disponibles. D'autre part, la possibilité de dupliquer les contenus à l'infini fait que l'informatique s'inscrit dès ses débuts dans une logique de diffusion massive et pratiquement sans frontières. Même avant l'avènement d'un Internet mondialisé, les logiciels circulaient vite et loin grâce à des réseaux de duplication parallèles. Si la famille de Christian achetait quelques jeux légalement au Radio Shack ${ }^{5}$ du coin, elle ne manquait pas de passer par le pusher de jeux copiés de Saint-Eustache à chaque visite à Montréal. I-Grec se rappelle que l'incontournable Radio Shack (celui de Granby cette fois) avait un concurrent: un "vieux monsieur de soixante-dix ans » avait ouvert un magasin d'informatique où on trouvait, bien en vue, une table de disquettes copiées à $5 \$$. En plus de ces sources plus ou moins officielles, les logiciels circulaient rapidement à travers les réseaux de cousins, d'amis de cour d'école, de gens fréquentant le même club informatique, etc. ${ }^{6}$ Ainsi, à l'instar des indies d'aujourd'hui, les jeunes Christian et I-Grec, malgré leur relatif isolement, pouvaient se tenir au fait de l'état des lieux du jeu d'ordinateur. Exposés à des jeux et à des logiciels qui les fascinent, Christian et I-Grec se découvrent le désir de faire leurs propres jeux.

\section{Apprendre à programmer}

Contrairement aux divers écrans contemporains qui sont conçus pour maximiser l'interaction intuitive, la première génération d'ordinateurs auxquels les jeunes Christian et I-Grec ont accès sont explicitement des machines qu'il faut programmer. Le TRS-80 n'a pas d'autre système d'exploitation que le langage de programmation BASIC. Alors qu'il suffit d'insérer une cartouche dans la console Atari 2600 pour qu'un jeu soit exécuté, il faut entrer une série de commandes pour charger et exécuter le moindre jeu sur cet ordinateur. Comme dit Christian, «c'était de facto quand t'achetais un ordinateur, t'allais programmer, sinon pourquoi tu l'achetais? » I-Grec pose une question rhétorique similaire : «Qu'est-ce que tu fais quand t'as un ordi chez vous? Tu veux le «driver $»^{7}$, tu veux comprendre comment ça marche. »

Aujourd'hui, les ressources pour apprendre à programmer (et faire des jeux informatiques en général) sont aisément disponibles - que ce soit sur Internet, dans les milieux scolaires ou par l'intermédiaire de clubs et d'organismes spécialisés. I-Grec et Christian ne disposaient d'aucune de ces ressources. D'autant plus qu'ils habitaient des villes de petite taille alors que l'informatique personnelle en était à ses balbutiements. Les deux ont alors entrepris de s'adonner à une pratique en voie de disparition : lire le manuel. Pour I-Grec, l'approche était simple : 
L'ordinateur venait avec deux disquettes : le DOS et GWBasic. Il y avait deux manuels : toutes les commandes DOS et toutes les commandes BASIC. « Faque ${ }^{8}$ je suis passé à travers les deux, [j'ai] essayé toutes les commandes DOS et toutes les commandes BASIC. Pis là tu comprends que c'est ça un ordinateur.

Il est intéressant de noter que l'école secondaire d'I-Grec avait proposé un cours de programmation qui, d'après le principal intéressé, a été un échec cuisant : « Ça a été un flop, il est pas revenu. Personne comprenait. Moi je trouvais ça ben cool.» Les deux créateurs rapportent que l'initiation à la programmation avait été facilitée par le logiciel LOGO, justement conçu à cette fin. «Ça faisait des lignes à l'écran, raconte I-Grec. On trouvait ça malade. »

Il est difficile pour les développeurs contemporains d'imaginer ce que signifie programmer un jeu à partir d'absolument rien. Les moteurs de jeu actuels (et presque tous les environnements de développement) offrent un très large éventail de fonctionnalités de base et l'on peut généralement se concentrer sur ce qu'un projet requiert de nouveau. Programmer sur un TRS80 signifiait que la plus banale des opérations, par exemple afficher un pixel, devait être effectuée manuellement. Alors pour tracer une ligne ou décoder un fichier d'image... D'ailleurs, Christian et I-Grec rapportent avoir dû programmer leur propre application de dessin pour tracer des images (à l'aide du clavier, la souris n'ayant pas encore fait son apparition) et les sauvegarder. Il faut les imaginer dans un long processus d'apprentissage, fait d'essais et d'erreurs, et de progressive sophistication au fur et à mesure qu'ils apprennent des techniques et enrichissent leurs propres bibliothèques de fonctions.

\section{Solitude}

Un des traits fondamentaux de phénomène indie contemporain est la constitution en communauté. Être développeur indépendant, c'est faire partie d'une famille qui se réunit à l'occasion de game jams, de festivals, mais ainsi que sur diverses plateformes en ligne (Juul, 2019, p. 141). Au Québec, on l'a vu, il existe même un organisme regroupant un grand nombre de développeurs : la Guilde du jeu vidéo. Au sein de ces communautés, les débutants peuvent trouver des outils d'apprentissage, du mentorat, profiter de commentaires ou de suggestions, etc.

Pour leur part, I-Grec et Christian s'initient à la conception de jeux dans un isolement presque complet. Si Christian a pu établir certaines collaborations pour l'audio et le visuel de ses jeux, son seul partenaire de programmation est son frère, qui partage son intérêt pour l'informatique. C'est lui qui l'aide à faire la transition vers le langage de programmation C. De son côté, I-Grec n'a jamais trouvé personne avec qui échanger sur cette passion. Le fait de vivre dans de petites villes de région, avant l'accès à Internet, n'a certainement pas aidé.

Si Internet n'a joué un rôle que vers la fin de leur parcours, dans la deuxième moitié des années 1990, l'accès aux bulletin board systems (BBS) ${ }^{9}$ constitue une étape importante pour les deux créateurs de jeux. Les BBS - ou babillards électroniques - agissent à la fois comme lieu de rencontre pour une communauté locale et comme pont vers le reste du monde de l'informatique. Puisqu'on y accède par la ligne téléphonique, les utilisateurs se rassemblent surtout sur les BBS locaux, s'évitant ainsi des frais d'interurbain. Ils agissent ainsi comme foyers pour l'établissement de communautés locales. Remarquons que c'est une chose qui a été 
perdue avec la migration vers Internet. Tout le monde ayant accès au même Internet, les communautés se forment désormais par concentration de champs d'intérêt - la langue exerçant un effet restrictif, bien sûr — plutôt que par proximité géographique.

Même lorsqu'ils visent un public local, les BBS deviennent des nœuds de distribution régionale au sein d'un réseau international. Si les logiciels circulaient déjà de manière fluide, les BBS ont encore accéléré ce processus. Christian raconte que plus d'une fois, alors qu'il téléversait son dernier jeu sur une série de BBS, il a découvert en arrivant au troisième ou au quatrième que le jeu y était déjà : quelqu'un l'avait devancé, ayant obtenu le jeu sur le premier BBS et téléversé sur l'autre entretemps. Pour le jeune Christian, qui souhaite vendre ses jeux, c'est le moyen tout indiqué pour maintenir une « veille »: «C'est là que ça t'informe sur le marché dans lequel t'évolues. J'ai joué à tout ce qui se faisait sur les BBS ». Pour I-Grec, c'est grâce aux BBS qu'il obtient les premiers retours sur ses jeux (en dehors de son frère et de ses amis proches) : «Le feedback que j'ai est comme fucking cool. Je distribue mon jeu, des gens jouent, c'est fucking cool. Le thrill ${ }^{10}$ est $\mathrm{p}$ [1] us juste pendant la création ». La circulation accrue ne concerne pas que les contenus, mais également les ressources de développement. Ni I-Grec ni Christian ne rapportent avoir pu utiliser les BBS comme une plateforme pour discuter programmation avec des pairs. Mais il s'agissait pour I-Grec d'une source d'outils :

Il commence à y avoir des librairies cools. Quelque chose pour charger un curseur de souris. Juste « loader ${ }^{11} \ldots$ Tu « loades » une image pis pas la voir se dessiner... «Fader ${ }^{12}$, ça a été un gros thrill! Voir que tu pouvais faire ça en BASIC !

En plus des BBS locaux, il y a certains grands BBS qui attirent une clientèle à l'échelle de l'Amérique du Nord. C'est le cas des BBS officiels de certaines grandes compagnies de jeux. IGrec et Christian rapportent d'ailleurs des mésaventures semblables quant aux frais d'appels interurbains. I-Grec raconte : « La première facture est arrivée. Pourtant je reste pas longtemps, une minute, deux minutes. Je sais que c'est des interurbains. Cinq cents piastres !!! » Christian, qui voulait diffuser ses jeux à grande échelle, ne peut éviter d'aller les téléverser sur de grands BBS tels que Software Creations, le BBS officiel d'Apogee : «J'avais pas beaucoup de temps pour faire autre chose qu' « uploader » mes trucs, on parle de centaines de piastres !»

\section{Oracle Software : aujourd'hui Amos, demain le monde}

\section{Faire de " vrais " jeux}

Après avoir conçu plusieurs petits jeux pour le plaisir et l'apprentissage, Christian publie son premier titre « sérieux » en 1989 : The Tournament of Zulula. À seulement quinze ans, il a déjà à peu près établi les paramètres de sa « manière ». Il s'agit d'un jeu de combat à deux joueurs dont Christian retrace ainsi l'inspiration :

[J]'avais fait un jeu sur TRS-80 appelé Sword Duel qui était un repeat du mode 2P de Trojan. The Tournament of Zulula était basé sur Sword Duel, mais avec une twist de tournoi d'élimination (inspirée directement du film The Karate Kid) et l'ajout de toutes sortes de monstres et créatures weird.

Voici un extrait de son texte promotionnel et quelques captures d'écran (figure 1). 
On the continent of Rastika, in the country of Zala, there lies the city of Zulula. A rich, prosperous city since the end of the Great War Of Freedom [...] The tournament of Zulula is an action-packed arcade game featuring multiple player character races (including odd creatures), single competition or tournament. ${ }^{13}$

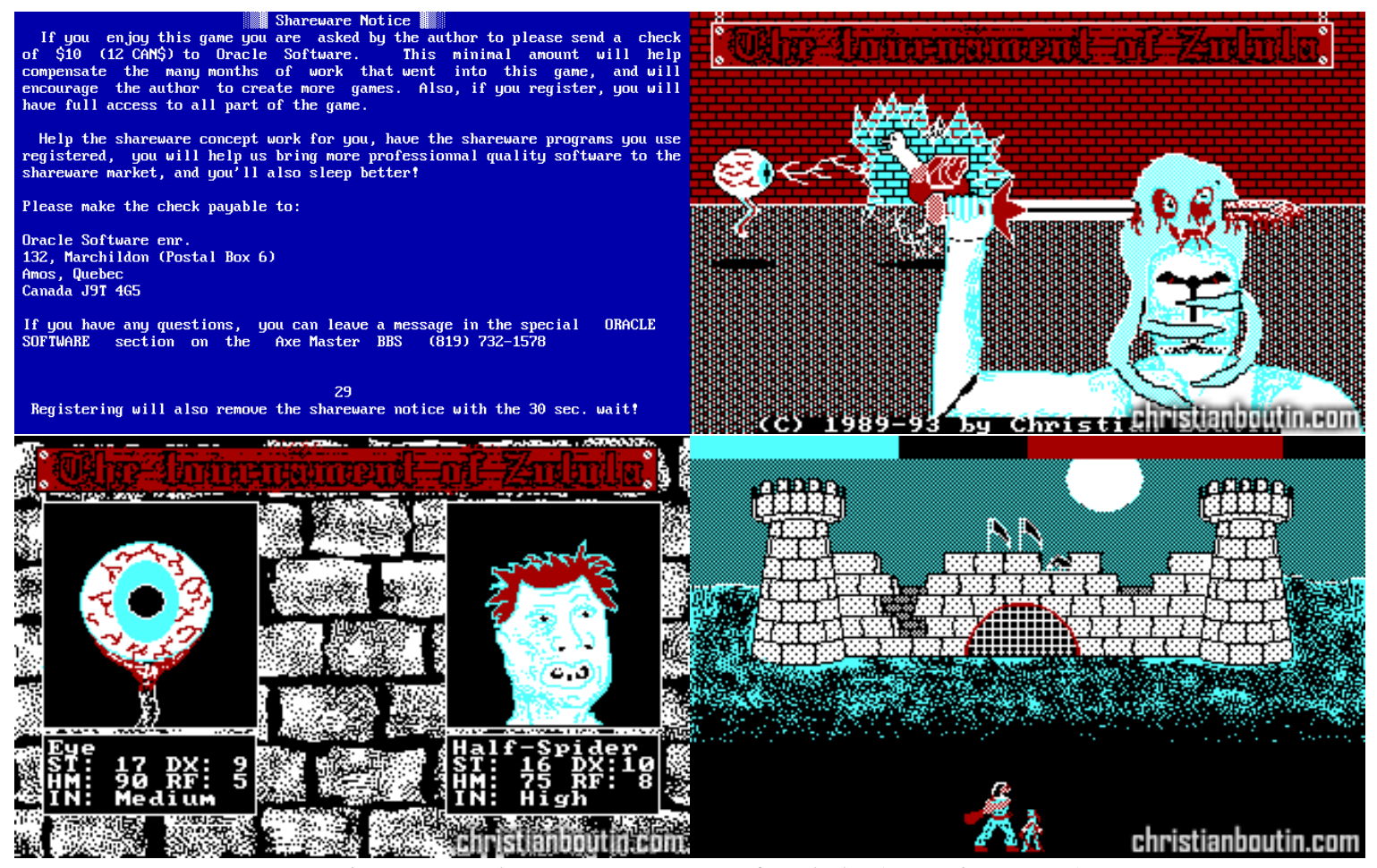

Figure 1. The Tournament of Zulula (Oracle, 1989).

On peut dès lors faire quelques observations spontanées : 1) le texte est en anglais ; 2) il y a un souci de détailler le monde fictionnel ; 3) le jeu s'inscrit dans un genre reconnaissable ; 4) la représentation graphique tente d'émuler l'état de l'art; et 5) le jeu est distribué en tant que partagiciel (shareware). En gros, Christian ne sort pas son jeu «pour le fun ». The Tournament of Zulula, et les titres suivants encore davantage, sont des propositions à placer aux côtés d'autres «vrais» jeux commerciaux. Son ambition est claire: vendre ses titres afin d'éventuellement pouvoir en vivre. Ses modèles sont les principaux éditeurs de jeux d'ordinateur du temps, mais tout particulièrement Apogee Software qui a popularisé le partagiciel comme mode de distribution ${ }^{14}$ :

J'avais tendance à me voir plus important que j'étais, je pensais que mes compétiteurs c'était Apogee. Dans ma tête, c'est là que je m'en allais. C'étaient des compagnies shareware parce que le scope était plus raisonnable.

\section{Une entreprise commerciale}

Lorsqu'on demande à Christian s'il se considérait comme un développeur indépendant, il rappelle que ce mot n'avait pas vraiment de sens à l'époque. Il se considérait comme un shareware developer. Cette posture orientera l'œuvre de Christian. De fait, elle trouve un écho dans une phrase répétée dans les manuels numériques accompagnant ses jeux : «Our goal is to 
bring more commercial quality programs to the shareware market ». On y retrouve la volonté de marquer un succès commercial, ainsi que la stratégie de ce succès : les mêmes genres de jeux que l'on trouve sur les tablettes du Radio Shack, mais au bas prix du partagiciel.

Le sérieux de la démarche de Christian se manifeste entre autres par l'enregistrement de sa compagnie Oracle Software en 1992, alors qu'il n'a que dix-huit ans. Ce n'est pas le nom qu'il avait choisi. Il s'est vu refuser Thunderstorm parce qu'au Québec on ne peut enregistrer un nom d'entreprise en anglais. Il cherche ainsi un mot qui fonctionne dans les deux langues. Il ne sait pas alors qu'Oracle Corporation est déjà une entreprise informatique majeure, laquelle lui enverra une mise en demeure deux ans plus tard. Il changera alors de nom pour OSP (Oracle Software Productions).

Pourquoi un jeune Amossois francophone du début des années 1990 conçoit-il des jeux en anglais ? Pour le Christian de l'époque, c'est une évidence :

Tous mes jeux de référence étaient en anglais. C'est sûr qu'il y avait une question de marché. À l'époque, rien n'était traduit. À l'époque, tout cet univers-là était en anglais. J'avais pas à me poser de question.

Comme aujourd'hui, l'anglais était la lingua franca de l'informatique et du monde du jeu vidéo. Il n'existait aucun modèle de jeu francophone ou québécois qu'il aurait pu émuler d'une quelconque façon. Faire un «vrai » jeu, pour Christian, c'est faire un jeu en anglais. À ce propos il ajoute :

Marché français comme dans la France? Non, pas vraiment. Honnêtement, mis à part quelques exceptions [...] les jeux vidéo semblaient être un truc vraiment centré sur les États-Unis [...] Donc, le marché américain était ma seule véritable cible. Ma première vente $[\ldots]$ était d'Allemagne, par contre. Mais ça a étrangement pas changé ma perspective.

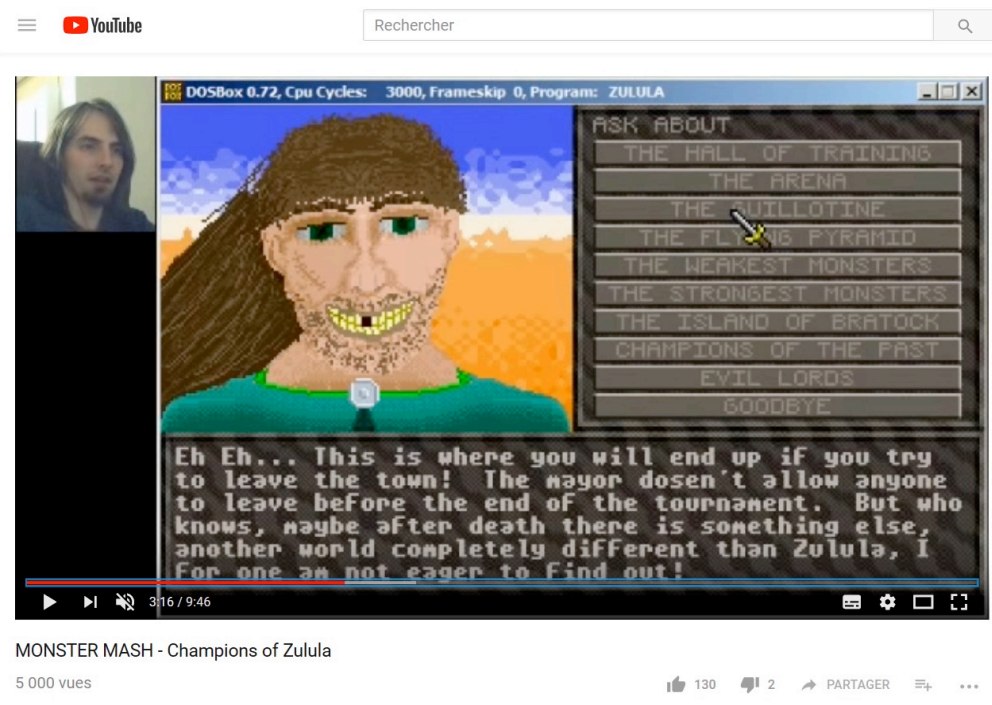

Figure 2. « Monster Mash - Champions of Zulula », par azuritereaction (YouTube, 2012). 


\section{Émuler les grands}

Nous l'avons mentionné, l'ambition d'Oracle était d'introduire des jeux de meilleure qualité sur le marché des partagiciels. Pour Christian, cela impliquait de reprendre des modèles phares du marché mainstream et d'en faire des versions aussi bonnes que possible quoique proposées à moindre coût. Il ciblait à la fois les genres qu'il appréciait, mais également ceux délaissés par les principaux joueurs du marché des partagiciels. «Mon public c'était: J'aime les jeux à la Ultima, mais bon, j'ai fini Ultima, alors quoi d'autre ? Justement, ses jeux les plus ambitieux - Walls of Bratock (1994) et la suite Flame Storm (1995) - sont fortement inspirés de la série Ultima (Origin Systems, 1981-2013), qui compte parmi ses jeux préférés.

Le choix du jeu de rôle à la Ultima est très ambitieux. Ce n'est pas pour rien que peu de développeurs de partagiciels s'y sont attaqués. Ces jeux sont à la fois complexes et de très grande envergure. Et Christian ne lésine sur rien, comme en fait foi le texte promotionnel :

Explore the HUGE Island of Bratock, visit more than 10 multi-level towns, villages and keeps, roam more than 30 HUGE dungeon levels, fight hordes of monsters, speak with dozens of people, all that in incredible 256-color VGA graphics, Sound Blaster music AND digitized sound effects. ${ }^{15}$

Christian y développe le monde fictionnel Akistar, dans lequel se situent aussi ses jeux d'action The Tournament of Zulula (1989) et Champions of Zulula (1995). Ce souci de richesse et de cohérence est caractéristique de l'ambition déclarée d'Oracle de faire des jeux de qualité. On ne se contente pas ici du minimum. Cette volonté de construire un monde fantastique détaillé, propice au déploiement de plusieurs trames narratives, témoigne aussi de sa longue fréquentation des jeux de rôle sur table et des jeux de guerre avec figurines.

Sans être tout à fait à la hauteur des jeux mainstream du moment, les graphismes rivalisent, somme toute, avec ce qu'on trouve sur le marché international des partagiciels. Christian s'assure de suivre l'évolution alors effrénée des exigences des joueurs. Il passe au mode graphique VGA à 256 couleurs dès Construction Bob in the Bouncing Factory, en 1993, et propose des cinématiques dans Walls of Bratock. Il utilise des images numérisées dans Flame Storm et même des animations générées en 3D dans Construction Bob Escapes from Hell (1995; figure 3).

\section{L'essoufflement}

Oracle atteint son pic commercial vers 1993, alors que les ventes de ses deux plus grands hits se chevauchent : Construction Bob in the Bouncing Factory et Walls of Bratock. Étudiant alors au cégep de Rouyn en informatique, Christian se demande : "Est-ce que j'y vais all-in ou pas? » Il choisit de foncer, retourne chez ses parents et se lance dans le développement de ses titres les plus ambitieux : le $3^{\mathrm{e}}$ épisode de la «saga de Bratock», ainsi qu'un jeu de stratégie guerrière fondé sur une licence d'une compagnie de figurines miniatures: Battlecry of the Reptiliads. Malheureusement, aucun de ces jeux ne verra le jour. 


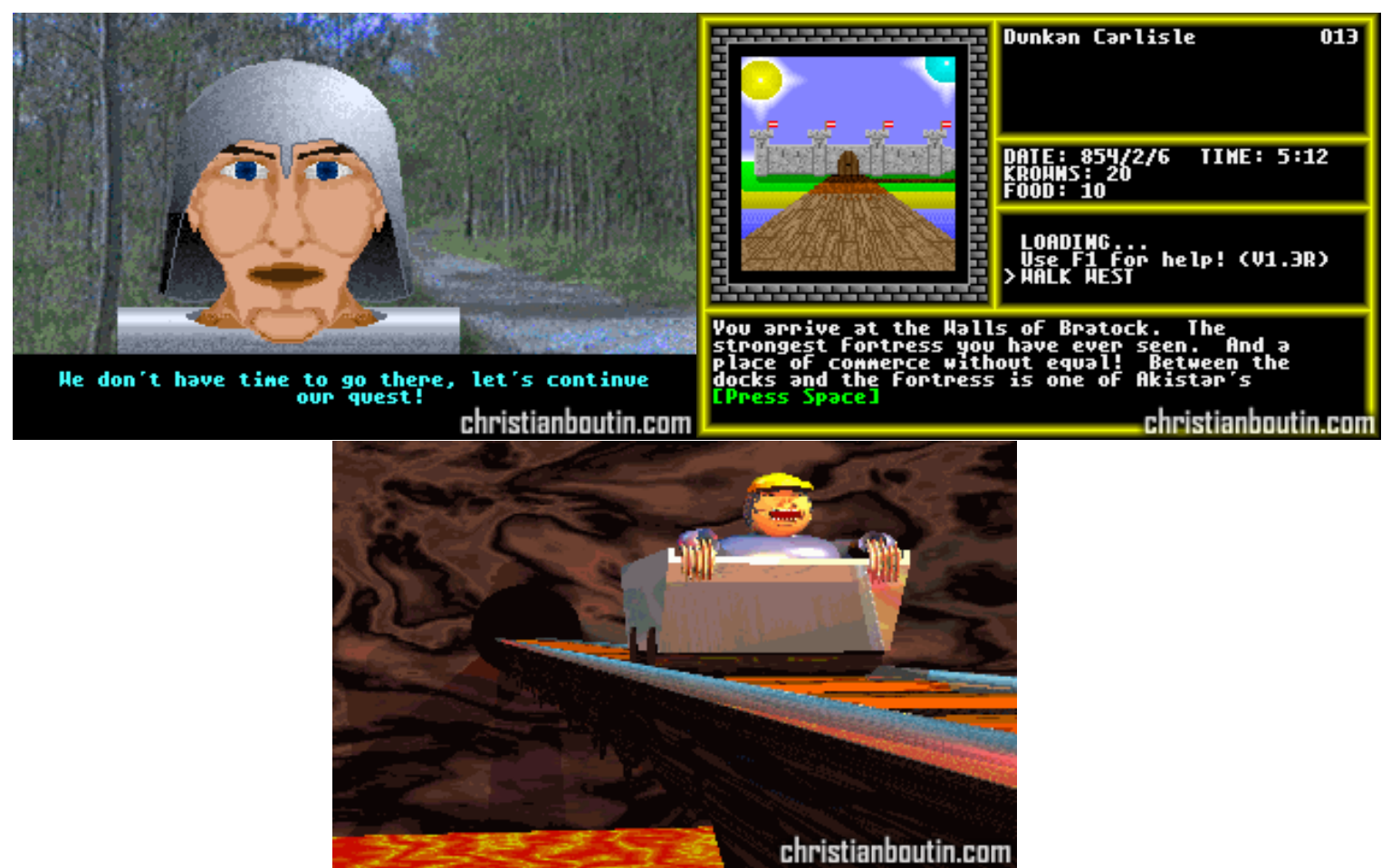

Figure 3. Éléments visuels tirés de Walls of Bratock (1994) en haut et de Construction Bob Escapes from Hell (1995) en bas.

C'était trop pesant, j'ai pas été capable. Il y avait de plus en plus de bogues. Le marché allait super vite. Les cédéroms entraient dans le portrait... J'avais commencé en VGA, tous les jeux étaient rendus en SVGA. J'ai tout « scrappé » ${ }^{16}$ à mi-chemin, je manquais de mémoire. Je suis passé à $\mathrm{C}++[\ldots]$ À un moment donné tu rames, tu rames, pis y'a des speedboats qui te dépassent. [...] J'ai atterri en burnout, à la fin des années 1990 .

Après trois ans d'efforts, Christian jette l'éponge. Il entrera éventuellement dans l'industrie « officielle» en obtenant un poste dans le nouveau service de jeu vidéo de Loto-Québec à Montréal. Il a depuis travaillé dans plusieurs compagnies de jeu, dont Hibernum, Triple Boris et Illogika.

C'est une histoire qui reste cruellement d'actualité. Les développeurs indie se lancent dans leurs projets avec passion. La plupart n'ont pas de modèle d'affaires réaliste et doivent éventuellement se tourner vers le travail salarié. Cet état de fait a d'ailleurs donné lieu dans les dernières années à de nombreuses conversations au sein de la communauté indie sur les moyens d'envisager une pratique "durable », qui n'impliquerait pas un inévitable épuisement à la fois personnel et financier (Juul, 2019, p. 121).

Il faut aussi mentionner que la période de l'abandon d'Oracle est particulièrement ingrate pour les développeurs indépendants. À ce moment, les attentes et les budgets ont amorcé une fulgurante ascension. Il est déjà à peu près impossible à une petite équipe de rivaliser avec les grandes productions. Le succès populaire de propositions innovantes, notamment avec la parution du célèbre DOOM (id Software, 1993), change la donne au point de vue du design 
comme de la technologie. L'idée de quelque chose qui soit «à peu près semblable, mais moins cher » n'enthousiasme pas le public. Sur ordinateur, ceux qui ne peuvent se payer les titres dominants les copient de toute façon. Finalement, à la fin des années 1990, il n'existe pas encore vraiment de sensibilité indie, pas de communauté encline à reconnaître la valeur d'un jeu malgré des moyens de production moindres. Il faudra attendre le milieu de la décennie suivante pour voir cette sensibilité émerger ${ }^{17}$.

\section{I-Grec : « dans le sens contraire depuis 1992 »}

\section{Faire des jeux pour soi}

Comme Christian, I-Grec commence en émulant ses modèles. Il est particulièrement inspiré par les jeux d'aventure textuels et graphiques. Plus personnelle, son approche est radicalement différente de celle d'Oracle qui tente d'emblée de développer des jeux pouvant s'inscrire dans le marché officiel des partagiciels. Le jeu le plus ancien parmi nos archives, F-MSC (19911992 ; figure 4) ${ }^{18}$, un jeu d'aventure graphique avec entrée de commandes textuelles, en est un exemple assez limite. L'introduction présente un récit à la première personne d'un étudiant de quatrième secondaire (l'âge d'I-Grec à l'époque) qui rêve de mettre le feu à l'école et de tuer tout le personnel enseignant. Le jeu invite justement le joueur à accomplir ce dessein. Le sujet morbide du titre est contrebalancé par un ton d'humour noir et un avertissement en début de jeu précisant qu'il s'agit d'une histoire fictive. Il n'en demeure pas moins que l'idée de faire un jeu offrant un point de vue sur une réalité personnelle - plutôt que de puiser dans les clichés des mondes fantastiques - a quelque chose d'original. Il faudra attendre les années 2010 pour que l'idée du jeu indie comme moyen d'expression fasse vraiment son chemin ${ }^{19}$.

Deuxième par ordre d'ancienneté dans nos archives, Dragon III : La légende de Zacharus ${ }^{20}$ (1992) est plus proche de ce qui caractérisera l'esprit I-Grec ${ }^{21}$. Il s'agit d'un autre jeu d'aventure graphique avec entrée de commandes textuelles récupérant les fonctions de programmation développées pour $F-M S C$. Le texte d'introduction vaut la peine d'être rapporté.

Il y a très longtemps, Zacharus vivait dans la ville de Death Land. Comme dans tous les jeux vidéo, il vivait en paix jusqu'au moment qu'arrive un monsieur pas gentil. Il hypnotisa tout le royaume sauf quelques exceptions comme : Jeanbon, Peracharle et Caporal twite [...]

Malgré son aspect parodique, cette prémisse annonce néanmoins un jeu substantiel. C'est là un des aspects de la signature d'I-Grec : prendre la peine de faire des jeux avec sérieux tout en adoptant une posture irrévérencieuse. Notons que les mises en abîme humoristiques étaient typiques des jeux d'aventure de la compagnie Sierra dont s'inspire I-Grec. Cette tradition du commentaire auto-référentiel sur le jeu vidéo s'était quelque peu perdue avant de revenir en force au sein de la production indie actuelle ${ }^{22}$.

I-Grec se permet cette liberté parce qu'il ne recherche pas le succès commercial ou critique. Il fabrique des jeux pour s'amuser, mais aussi pour voir ce dont il est capable. C'est également ce qui justifie son choix du français comme langue : il fait des jeux pour lui, son frère, quelques amis peut-être, et éventuellement les BBS locaux. C'est un monde en français, mais pas n'importe quel français ; I-Grec choisit d'écrire comme parlent les jeunes de son âge : 


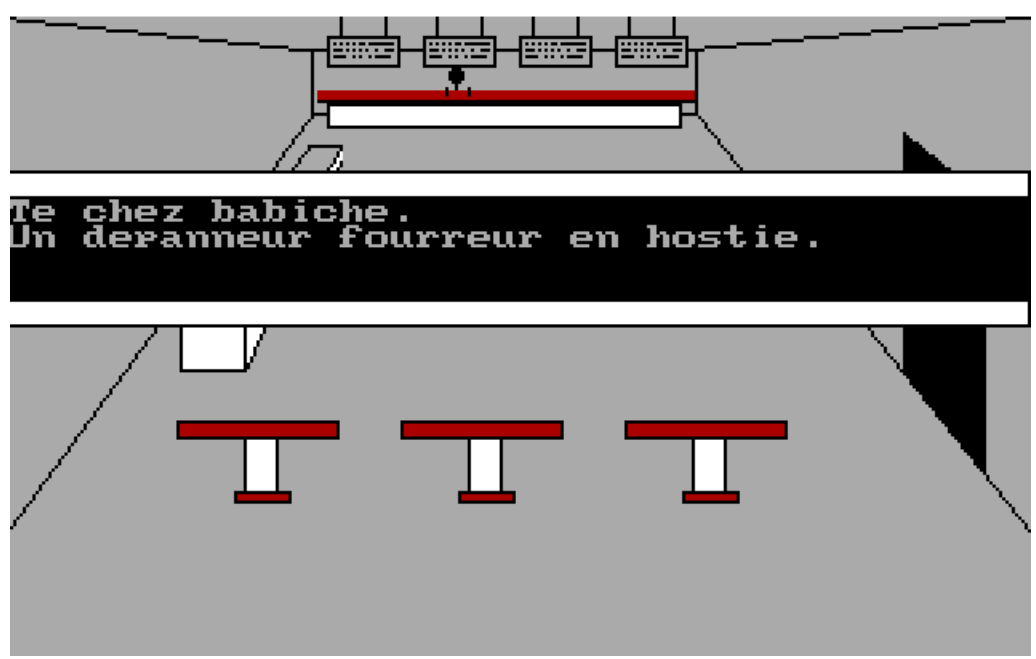

Figure 4. F-MSC ${ }^{23}$ (I-Grec, 1991-1992).

C'est comme ça qu'on parlait pis c'est comme ça qu'on chattait sur les BBS. Tu vas pas mettre des vrais verbes sinon c'est comme si t'es un adulte. T'aurais eu l'air vraiment minable d'essayer de faire une affaire comme Sierra avec de la marde comme ça.

I-Grec adopte une posture d'authenticité qui évoque ce que Jesper Juul observe dans le phénomène indie actuel (Juul, 2014). Il s'agit d'être " vrai », que le jeu reflète la sensibilité de son auteur plutôt que d'être taillé à la mesure des études de marché. Plus de vingt-cinq ans plus tard, l'attitude d'I-Grec est parfaitement compatible avec le manifeste du collectif de développeurs de jeux montréalais FLOP, en particulier avec la série de propositions ci-dessous (voir le texte entier à la figure 5) :

Crée des jeux pour toi-même. [...] Crée quelque chose, n’importe quoi. [...] Oublie le succès. Le marché est déjà saturé, entendras-tu. Ce que tu crées n'a pas à appartenir à qui que ce soit. L'important est qu'il t'appartienne. Une menue fenêtre vers ta personne.

I-Grec admet que le choix du français était également conditionné par sa connaissance approximative de l'anglais. Il savait aussi qu'il y avait là quelque chose d'unique et de remarquable : «J'étais conscient que c'était fucking cool d'avoir un jeu qui parle comme ça. On va le faire comme on parle nous autres. » C'est là une autre caractéristique de la méthode d'IGrec : exploiter ses limites pour faire quelque chose de différent. Comment ne pas sourire lorsqu'on découvre les boutons-verbes de son jeu d'aventure Tabarnak (1996 ; figure 6) ?

\section{Bricoler du neuf}

I-Grec est parfaitement conscient de ses limites comme concepteur de jeux d'ordinateur : «Je suis pas bon en dessin, je suis pas capable de faire des beaux dessins. Tabarnak, c'est un peu dégueu, mais je suis pas capable de faire mieux. J'essayerai pas. » Il décide donc de viser une esthétique brute tout en s'assurant de bien en souligner l'aspect volontaire : 


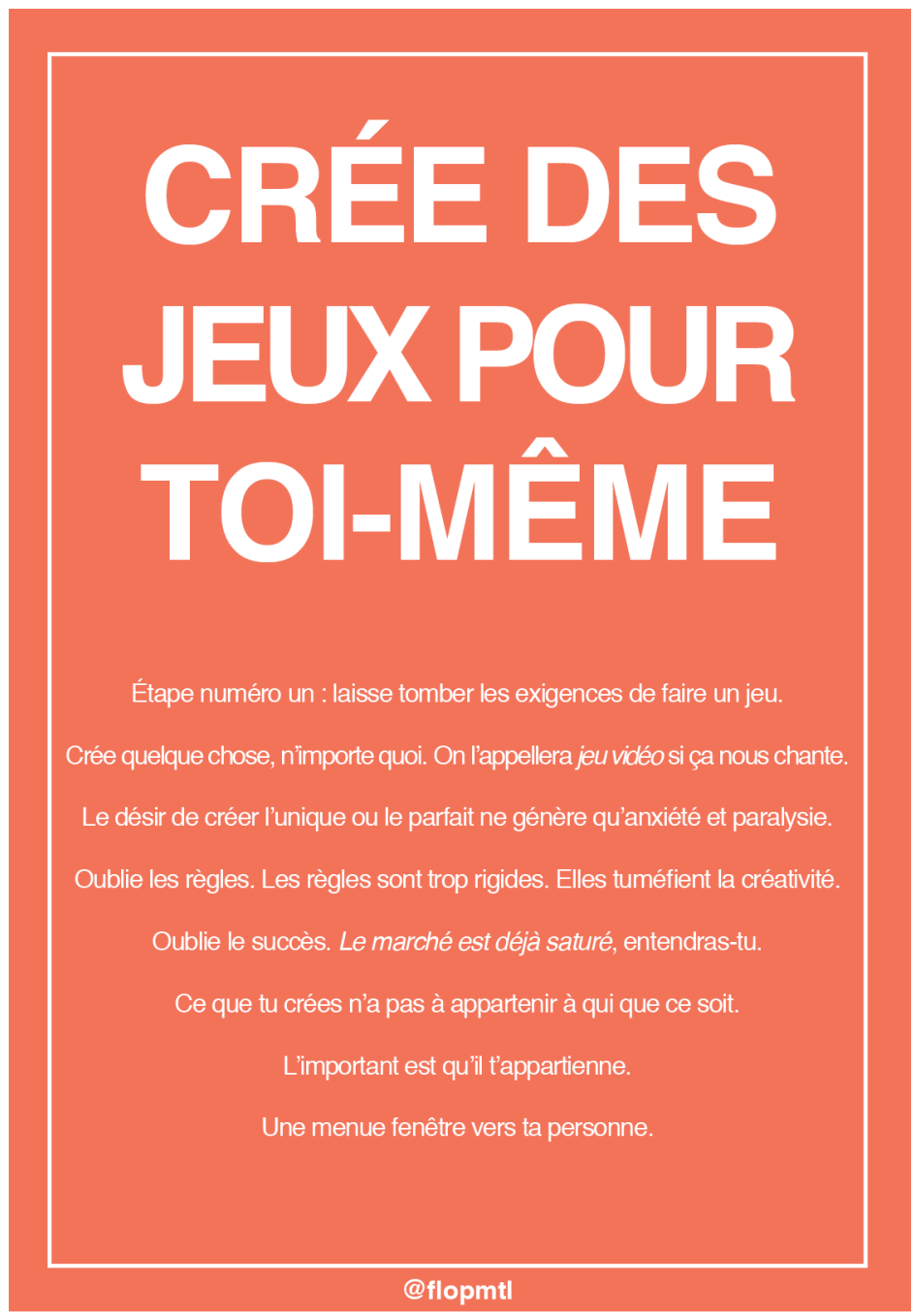

Figure 5. Poster du collectif de jeux vidéo montréalais FLOP (2018).

Comment je peux le rendre intéressant? Faire ça vraiment garage. Ça va faire partie de l'esthétique du jeu. C'est pas beau, mais c'est le but que ce soit pas beau.

C'est la même stratégie que Jesper Juul observe chez les indies contemporains. Ce qu'il appelle le "independent style » repose sur un style visuel volontairement moins sophistiqué que la norme des jeux grand public (le style pixélisé dit 8 bits, par exemple), mais qui — et c'est là l'aspect important — participe de l'authenticité du jeu plutôt que d'être perçu comme un manque, comme une incapacité à faire mieux (Juul, 2014). Même si souvent, comme c'est le cas d'I-Grec, cette incapacité est tout aussi réelle. Notons que l'esthétique trash assumée de ce dernier reste cependant beaucoup plus rare que l'exploitation d'un style rétro.

Sur le plan visuel, I-Grec adopte deux procédés. Le premier est le même que plusieurs indies actuels, soit l'utilisation volontaire d'une très basse résolution permettant des illustrations de type pixel art (nous reviendrons plus tard sur les autres motivations de cette approche). L'autre, 


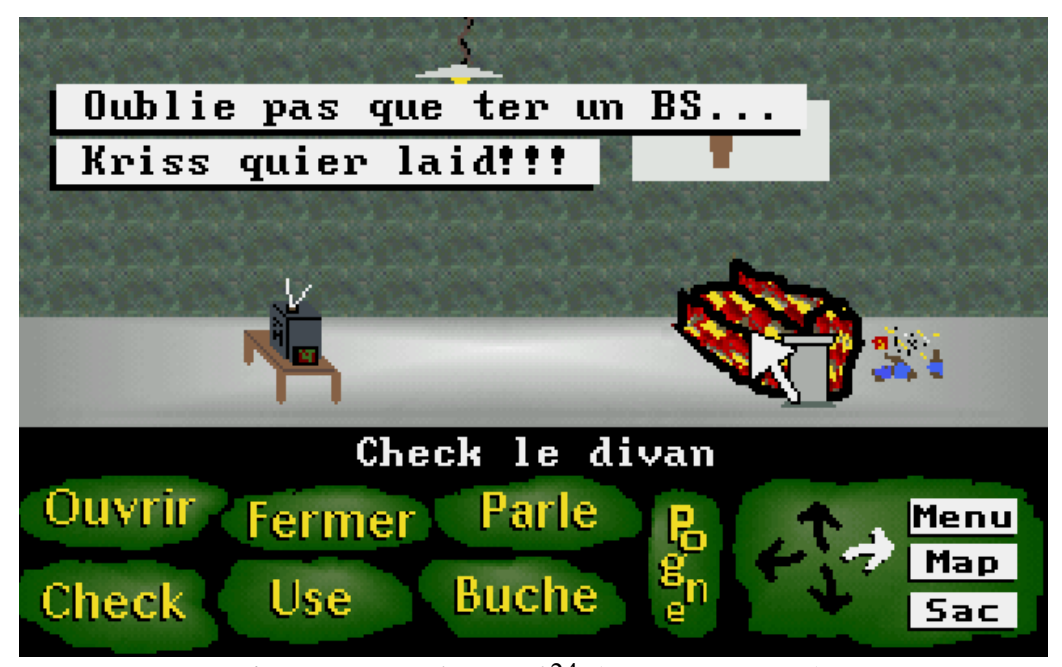

Figure 6. Tabarnak ${ }^{24}$ (I-Grec, 1996).

qu'il utilise abondamment dans Tabarnak, consiste en des collages d'images digitalisées grappillées ici et là. "Les images scannées, c'était rare! Quand t'en trouvais une, tu la gardais ! » Ainsi, dans Tabarnak, l'apparition de Jacques Parizeau dans le rôle d'Ostie d'Kriss (figure 7) est, selon lui, le fruit du hasard plutôt que d'une volonté de faire un commentaire politique.

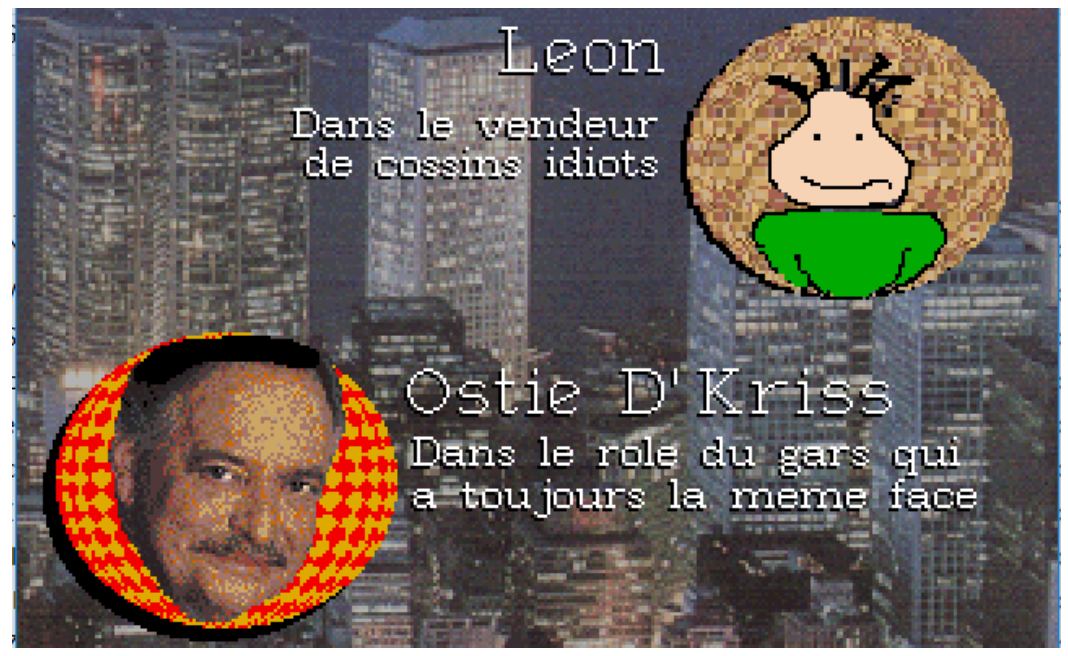

Figure 7. Tabarnak (I-Grec, 1996).

L'autre aspect qui compte, pour I-Grec, c'est de faire quelque chose de nouveau. C'est là où sa démarche s'écarte radicalement de celle d'Oracle et qui vise plutôt l'émulation.

En 1995, tout a été fait. Tous les estis de types de jeux ont été faits. Tous les concepts de jeu ont été faits. C'était un peu ça, mon challenge. J'ai aucun intérêt à passer deux ans de ma vie à programmer, passer des nuits blanches, pour un autre jeu pareil. Je voulais trouver quelque chose de différent, c'est pour ça que j'avais des concepts crackhead. Il y a aucun concept comme ça. "I-Grec : dans le sens contraire depuis $1992 »$, c'est un peu ma vie, j'ai tout le temps été comme ça. Si tout le monde fait ça, je vais me forcer pour trouver un autre chemin même si c'est dur. 
Cette recherche de la différence radicale est manifeste dans à peu près tous les jeux d'I-Grec. Mentionnons le provocateur Masturb Joe in Alpha Zone (1998), qui met en scène un homme nu dont le désir sexuel toujours croissant doit être assouvi par une masturbation inlassable. Ce soulagement est cependant compliqué par un sentiment de " gêne » tout aussi croissant lorsqu'il est pratiqué loin de l'arbre qui ménage une certaine intimité. Sauf que des pommes peuvent tomber de l'arbre et assommer le pauvre homme. Finalement, la masturbation empêche l'avatar de se déplacer pour aller chercher les objets qui donnent des points mais qui, bien évidemment, apparaissent loin de l'arbre (figure 8).

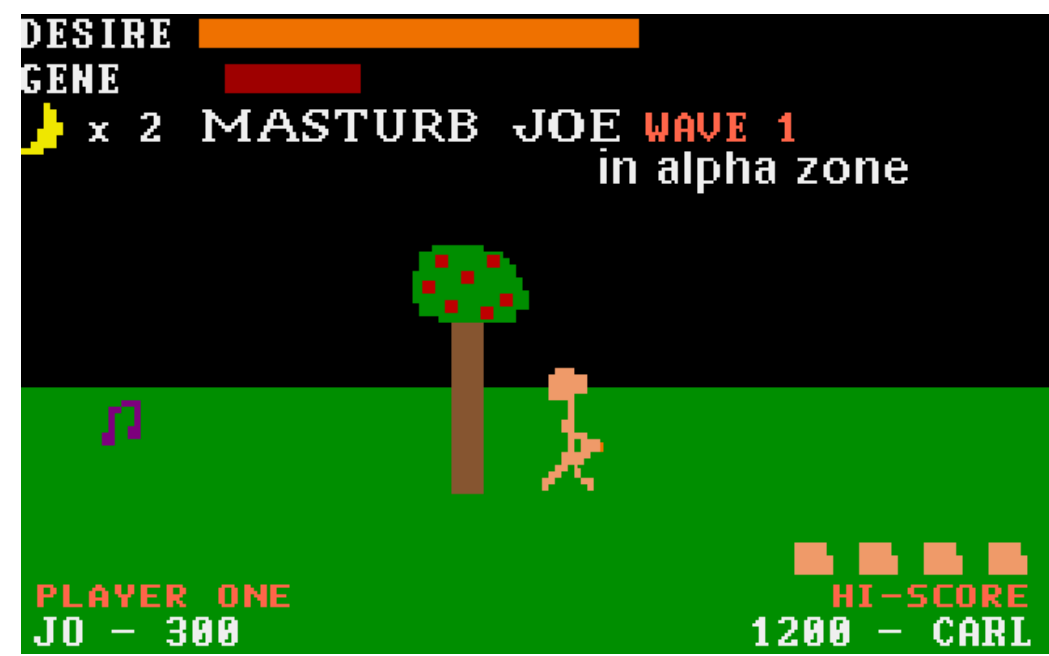

Figure 8. Masturb Joe in Alpha Zone (I-Grec, 1998).

On peut pareillement mentionner brièvement Bruno : Spécial Mexique (1999), jeu qui montre l'animateur de télévision Bruno Blanchet tenter d'attraper, à l'aide de sa longue langue, des nachos qui tombent du ciel. Il s'agit là, à notre connaissance, du seul jeu vidéo mettant en scène un représentant du star système québécois !

\section{Retour aux sources}

Dans la deuxième moitié des années 1990, I-Grec redécouvre les premiers jeux vidéo Atari 2600 et ColecoVision par l'intermédiaire des émulateurs qui font alors leur apparition. Rappelons que ses parents avaient toujours refusé de laisser entrer les consoles de jeu dans la maison familiale. C'est l'occasion pour I-Grec de renouer avec une fascination d'enfance :

J'ai toujours rêvé dans ma jeunesse à avoir un Atari pis un Nintendo, mais c'était interdit. Mes amis en avaient, je trouvais ça écoeurant. La manette, tu diriges la TV. C'est le futur!

I-Grec tombe sous le charme de la simplicité, du mystère et de l'efficacité de ces jeux que d'autres pourraient considérer comme rudimentaires :

Les jeux Atari, c'est beau, c'est de toute beauté. C'est juste des gros pixels pis c'est l'fun. C'était écoeurant, ça me touchait. Ce que je trouvais fascinant, c'est que c'est pas une reproduction de la réalité, c'est quelque chose de complètement différent. 
[...] Un jeu Atari, le fond est noir pis y'a un carré qui bouge. C'est... L'imagination fait boum.

À partir de ce moment, la plupart des jeux d'I-Grec prennent la forme d'hommage aux jeux de cette époque (tout en conservant le ton et les motifs typiques de son œuvre). Cela transparait clairement dans la direction artistique (comme on peut le voir dans Masturb Joe), mais également dans le design. Masturb Joe et Tuque Rose (1999) s'éloignent du modèle du jeu d'aventure pour s'engager dans la voie des mécaniques simples et efficaces des premiers jeux de console : sauter, éviter, attraper, etc. I-Grec est aussi fasciné par le caractère mystérieux de certains jeux comme E.T. (1982) qui, en raison de leurs illustrations presque abstraites, en disent très peu sur la nature des lieux, des personnages et de l'action : "C'est toute la beauté des backgrounds, tu sais pas c'est quoi, c'est fascinant ! C'est superbe. [...] Tu sais pas ce qui se passe. »

Cette inspiration culmine avec le dernier jeu d'I-Grec, UI (1999), qui se présente comme un pur mystère à percer. Le jeu évoque un peu E.T., justement, ou le premier Zelda dans sa représentation : un personnage à la tuque rose, vu du haut, qui peut passer d'une scène à l'autre. La figure 9 montre les seules instructions d'UI.

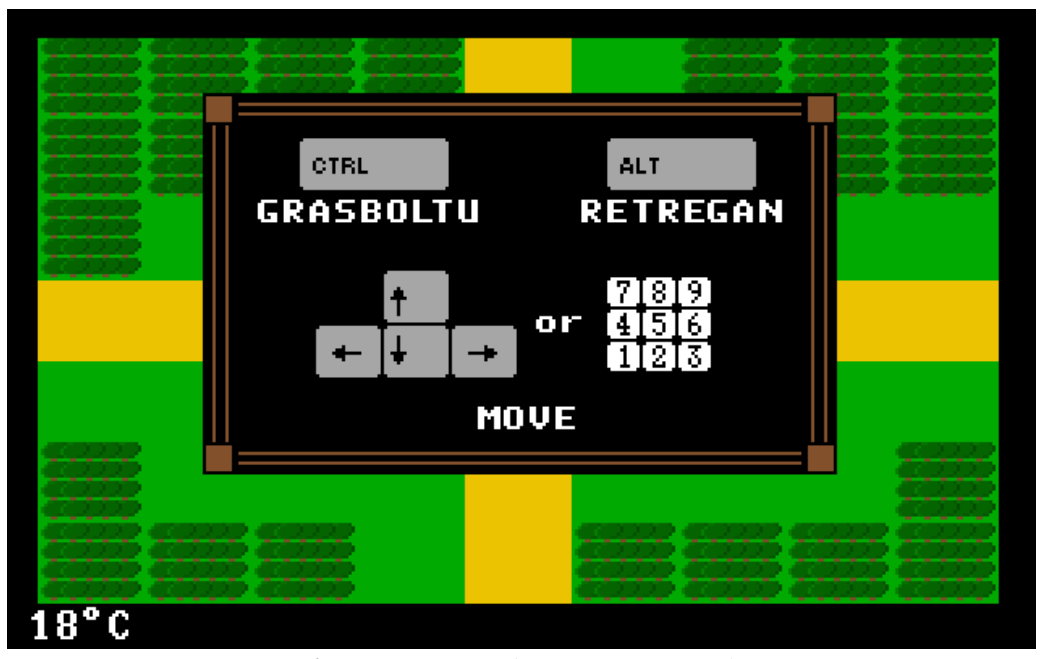

Figure 9. UI (I-Grec, 1999).

Le joueur dispose donc de deux commandes cryptiques : GRASBOLTU et RETREGAN. Rien n'indique leur fonction, et rien de sensible ne se passe lorsqu'on les active. Il faut supposer qu'elles agiront dans un contexte précis, mais lequel ? Autre mystère, l'écran indique une température : 18 degrés. Faut-il la faire changer? Comment? Pourquoi ?

Il s'agit d'une vraie rupture avec la tendance forte du jeu de l'époque (et encore plus de nos jours) visant à sécuriser au maximum le joueur en lui communiquant tous les paramètres nécessaires à la compréhension du jeu. Il faudra attendre encore les années 2010 pour voir apparaître des jeux indie qui embrassent ainsi le mystère et fondent leur expérience sur la découverte progressive des paramètres du monde ${ }^{25}$. On peut penser, par exemple, à Proteus (Kanaga et Key, 2013), qui a lancé la mode des «simulateurs de marche» (walking 
simulators), des jeux d'exploration libre fondés sur le mystère et la contemplation plutôt que sur le défi ludique.

\section{Désenchantement}

Lorsqu'on lui demande pourquoi il n'a pas continué à faire des jeux, I-Grec évoque un certain désenchantement :

Quand j'étais petit, j'étais sûr que j'allais travailler dans les ordis, mais après...

Windows... Shockwave... Bleh... C'était pas ça le futur. J'avais pas beaucoup d'intérêt pour ça. Ça faisait plus me fâcher que d'autre chose.

La redécouverte des premiers jeux vidéo ne fait qu'exacerber le dégoût qu'inspire à I-Grec l'évolution de l'informatique, et dont la manifestation la plus irritante est le système d'exploitation Windows.

J'avais parti un mouvement «Fuck Windows!» Vingt megs pour « runner» Windows 3... Qu'est-ce qui a là-dedans qui prenait $20 \mathrm{MB}$ ? T'as le DOS qui prend genre $20 \mathrm{~K}$ de mémoire vive pis Windows qui demande un meg. J'étais convaincu que c'était une crosse, une arnaque pour vendre des ordis plus forts. La seule raison pourquoi tu dois changer ton ordi, c'est pour faire rouler Windows, pour faire rouler l'économie.

Windows est l'incarnation de deux tendances qui irritent I-Grec: la marchandisation de l'informatique et, ironiquement, sa démocratisation. Contrairement aux consoles de jeux vidéo qui ont toujours à peu près réussi à protéger leurs marchés et à forcer les utilisateurs à payer pour les cartouches, l'informatique des premiers temps est une sorte de monde libre, repère de pirates où tout est gratuit dans les faits puisque tout se copie librement. Son mépris de la marchandisation s'étend même aux créateurs de jeux :

Man, tu demandes vingt piastres pour tes jeux ? Tu fais pas ça parce que t'aimes ça ? On le copie de toute façon, t'as juste l'air cave. C'est de même que ça fonctionnait, tout était gratuit. Sur le terrain, tu copiais des jeux. T'achetais pas le DOS, tu le copiais. Tu savais pas qui qui payait ça.

I-Grec manifeste cette prise de position dans ses jeux et même son logo (figure 10). On peut lire dans le texte déroulant de la page d'accueil de Bruno: «Supportez les programmeurs anticapitalistes !»

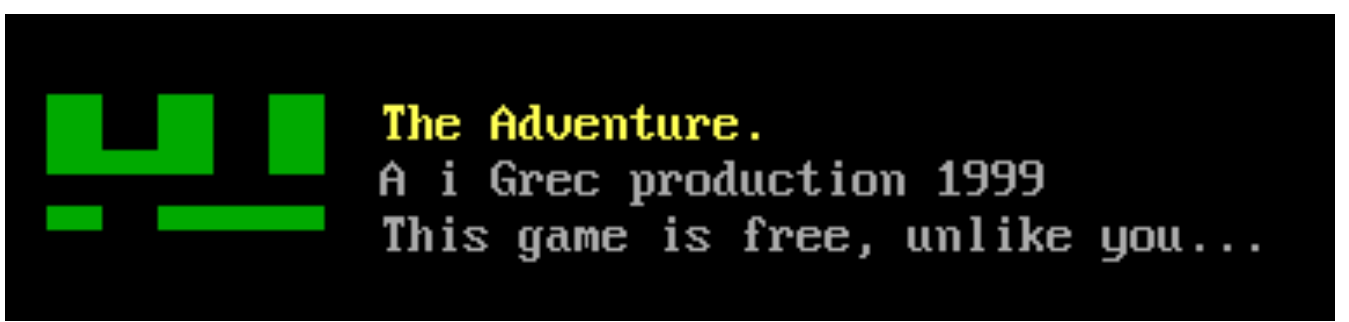

Figure 10. Logo d'I-Grec. 
Cette position radicale trouve moins d'écho dans le mouvement indie actuel. Comme l'observe Juul, l'éthique d'authenticité du mouvement s'accommode de la nécessité de devoir gagner sa vie en vendant ses produits et évoque plutôt «l'entrepreneuriat bohême » que la mouvance punk (2019, p. 24). I-Grec peut quand même compter quelques héritiers spirituels, comme le développeur Molleindustria dont les jeux gratuits constituent une critique forte du capitalisme et de l'impérialisme.

Windows incarne un autre mal pour I-Grec, celui de simplifier l'informatique pour la rendre accessible à tous :

Windows, c'est pour que les mononcles et les matantes achètent des ordis. Nous autres on sait comment ça marche, c'est pas pour les mononcles, c'est pas pour les matantes.

C'est comme si I-Grec se voyait dépossédé d'une part de sa rébellion contre le monde. Les ordinateurs représentaient un aspect d'une certaine culture jeune et rebelle qui allait être gâchée si tout le monde s'y mettait.

I-Grec se sent également en décalage avec l'évolution esthétique et technologiques des jeux vidéo. Alors qu'il redécouvre la simplicité et l'élégance des jeux Atari, la tendance est plutôt aux cédéroms et aux films interactifs :

Le jeu vidéo, c'est électronique. Il y a un espace entre l'utilisateur et la télé, mais tu peux rentrer dedans avec ta manette. Pour moi c'était ça le futur, créer quelque chose de nouveau. Pas recréer des belles images de château médiéval. La télé fait bien ça. [...] Y'ont sorti le cédérom : 500 megs pis c'est fucking platte ! [...] On dirait que la transition a pas été très artistique.

Résultat : I-Grec décroche et déclare ne pas avoir joué à un nouveau jeu depuis King's Quest V (1990). Son élan créatif ne s'essoufflera pas pour autant. Quelques années plus tard, il fonde le groupe de musique «Le volume était au maximum » avec lequel il sortira quinze albums en quinze ans.

\section{Et le Québec ?}

Les œuvres de Christian et I-Grec auraient-elles été différentes s'ils provenaient de petites villes américaines ou européennes? Sans doute. Il est bien sûr difficile de tirer la moindre généralisation d'un échantillon si petit, mais on peut au moins remarquer la différence radicale entre leurs attitudes. En tant que média nativement numérique et mondialisé dès son émergence, le jeu vidéo laisse la question du public entièrement libre. C'est à chacun de choisir à qui il s'adresse. Oracle cible le monde (typiquement, cela veut surtout dire les États-Unis), ce qui l'amène à écrire en anglais et à puiser dans un fonds culturel plus ou moins commun (le fantasy, la science-fiction). Quant à I-Grec, il s'adresse surtout à lui-même, et écrit donc dans sa propre langue (allant jusqu'au vernaculaire régional), et il se permet d'emprunter à sa culture locale sans se soucier que le « monde » puisse ne pas comprendre. 
On aura remarqué au fil du texte qu'on trouve déjà chez Christian et I-Grec plusieurs attitudes et approches caractéristiques du mouvement indie actuel. Cette observation suggère que le phénomène n'est pas que lié à l'émergence de nouvelles pratiques ou d'une génération de créateurs géniaux, mais doit beaucoup à un changement de contexte culturel, à la maturation d'un public prêt à accueillir et à valoriser un jeu vidéo différent. On peut cependant regretter que si les ambitions esthétiques d'I-Grec trouvent aujourd'hui un large écho, peu d'artistes prennent le parti de mettre en valeur la culture locale. Dans un marché aussi saturé que celui du jeu vidéo, au sein duquel un jeu indépendant a très peu de chances d'être remarqué parmi la masse publiée chaque jour, il y a fort à parier qu'un jeu intitulé Tabarnak trouverait immédiatement un nombre appréciable de curieux au Québec.

\section{Remerciements}

Les auteurs tiennent à remercier Bernard Perron pour sa rigueur et sa remarquable ténacité, les évaluateurs anonymes pour leurs commentaires constructifs et Odette Provost pour ses attentionnées révisions linguistiques.

\section{Références}

Juul, J. (2014). High-tech Low-tech Authenticity: The Creation of Independent Style at the Independent Games Festival. In Proceedings of the 9th International Conference on the Foundations of Digital Games. Fort Lauderdale. En ligne: https://ieeexplore.ieee.org/document/8951050.

Juul, J. (2019). Handmade Pixels: Independent Video Games and the Quest for Authenticity. Cambridge (Mass.), The MIT Press.

Parker, F. et J. Jenson (2017). Canadian Indie Games Between the Global and the Local. In Canadian Journal of Communication 42(5). En ligne: https:/www.cjconline.ca/index.php/journal/article/view/3229.

PC Gamer (2006). The Twelfth Annual PC Gamer Awards. 13(3), 33-44.

Sidre, C. (2015). Du rôle des sociétés de distribution et des boutiques de micro-informatique dans la naissance de l'industrie vidéoludique française. In Kinephanos (numéro spécial "Histoire culturelle du jeu vidéo"). En ligne : https://www.kinephanos.ca/2015/naissanceindustrie-videoludique-francaise/.

Swalwell, M. (2008).1980s Home Coding: The Art of Amateur Programming », in S. Brennan et S. Ballard. Aotearoa (dirs.), The Aotearoa Digital Arts Reader (p. 193-201). Aotearoa Digital Arts and Clouds.

\footnotetext{
${ }^{1}$ Notons que les jeux dits indépendants sont en grande majorité parfaitement intégrés aux réseaux commerciaux existants et qu'à cet égard leur « contestation » porte surtout sur des enjeux esthétiques.

${ }^{2}$ Il s'agit ici de l'Independent Games Festival associé à la Game Developers Conference.

3 La Guilde, «La plus grosse coopérative de jeux vidéo indépendants au monde voit le jour au Québec », communiqué de presse du 5 avril 2016, https://www.laguilde.quebec/wpcontent/uploads/2016/04/Communique-La-Guilde.pdf.
} 
${ }^{4}$ Il existe quelques exceptions notables au Québec, comme Sang-Froid: Tales of Werewolves (Artifice Studio, 2013), qui puise dans le folklore québécois, et Kona (Parabole, 2016), qui se situe explicitement dans le nord du Québec des années 1970.

${ }^{5} \mathrm{Au}$ Québec ainsi que dans plusieurs régions en Amérique du Nord, la chaîne Radio Shack était souvent le principal (ou l'unique) détaillant grand public de produits informatiques.

${ }^{6}$ Colin Sidre observe un comportement analogue en France: le Centre mondial de l'informatique, lieu qui rassemble les amateurs, a été nommé « centre mondial de piratage » par le magazine Hebdogiciel (2015).

${ }^{7}$ « Driver » un ordinateur : le pousser à bout.

8 «Faque » : donc.

${ }^{9}$ Un bulletin board system (une technologie à la disposition du grand public depuis le début des années 1980) est un programme exécuté sur un ordinateur connecté au réseau téléphonique par un modem. Les utilisateurs (disposant d'un ordinateur avec modem aussi) pouvaient s'y connecter chacun son tour (à moins que le BBS ne dispose de plusieurs lignes téléphoniques) pour y télécharger des fichiers, écrire des messages sur des forums et s'adonner à certains jeux asynchrones multijoueurs.

${ }^{10}$ Thrill : frisson.

11 « Loader» : charger.

12 «Fader » : faire un fondu au noir.

${ }^{13}$ Sur le continent de Rastika, dans le pays de Zala, se trouve la ville de Zulula, une riche ville prospérant depuis la fin de la Grande Guerre de la Liberté [...] Le tournoi de Zulula est un jeu d'arcade riche en action, permettant de choisir entre des personnages de diverses races (incluant d'étranges créatures), un mode de compétition simple ou de tournoi. [Notre traduction.]

${ }^{14}$ Apogee Software sera particulièrement reconnu pour la publication des best-sellers Commander Keen (1990) et Wolfenstein $3 D(1991)$.

${ }^{15}$ Explorez l'immense île de Bratock, visitez plus de dix villes, villages et donjons multiniveaux, errez dans plus de trente fabuleux niveaux de souterrains, combattez des hordes de monstres, conversez avec des douzaines de personnes, et tout ça dans un environnement incroyable, avec son graphisme VGA en mode 256 couleurs, sa musique Sound Blaster et ses effets sonores numériques. [Notre traduction.]

16 « Scrapper » : mettre au rebut.

${ }^{17}$ On peut évoquer Darwinia (Introversion, 2005), par exemple, qui gagne l'Independent Games Festival (de la Game Developers Conference) et dont la revue PC Gamer (2006) souligne l'esthétique retro-cool.

${ }^{18}$ Le sigle $F-M S C$ signifie littéralement « Fuck, Mont-Sacré-Cœur », le nom de l'école secondaire d'I-Grec.

${ }^{19}$ Pensons à Dys4ia, d'Anna Anthropy, qui met en scène sous forme vidéoludique le processus de réassignation de genre de l'autrice.

${ }^{20}$ Dragon $I$ et $I I$ ont malheureusement été perdus.

${ }^{21}$ Notons que les jeux sont publiés sous le nom de Peyeb Games, puis de Tabarnak Games. C'est à partir d'Ö̈ (1996) que l'étiquette I-Grec est adoptée pour de bon.

${ }^{22}$ Juul parle d'une approche « authentiquement méta » (2019, p. 156).

${ }^{23}$ " [Tu es] chez [B]abiche. Un dépanneur [très arnaqueur]. »

${ }^{24}$ « [N']oublie pas que [tu es] un [assisté social]. Il est vraiment très laid !!! »

${ }^{25}$ Juul parle d'une approche « authentiquement opaque » (2019, p. 150). 